\title{
LA EDUCACIÓN A DISTANCIA EN LAS UNIVERSIDADES DE AMÉRICA LATINA (Avance de Investigación)
}

\author{
YOLANDA BUSTAMANTE SÁNCHEZ* \\ E-mail: yobusalhotmail.com
}

\begin{abstract}
RESUMEN
El objetivo del presente avance de investigación es el de analizar la realidad de la educación a distancia en diversas universidades de América Latina. Para ello observaremos su FODA institucional con el cual evaluaremos, compararemos y contrastaremos la educación en línea versus la educación tradicional, buscando i dentificar las bases de una educación de calidad que se puedan aplicar en las universidades de nuestro país.
\end{abstract}

Palabras clave: Educación a distancia, educación on-line, proceso de enseñanza-aprendizaje.

\section{ABSTRACT}

The objective of this research is to analyze the reality of long-distance education in different universities of Latin America. For this purpose we will observe its Institutional FODA in order to evaluate, compare and contrast on-line education vs. traditional education trying to identify the bases of education quality that can be applied on universities of our country.

Keywords: Long distance education, on-line education, process of teaching-learning.

\footnotetext{
Licenciada en Educación. Miembro fundador de Red Peruana de Asistencia y Desarrollo (REPAD) y Coordinadora de Relaciones Públicas de dicha institución.
} 
Si nos remontamos a los inicios, la modalidad a distancia nace de las urgencias impuestas por el desarrollo industrial; urgencias de entrenamiento en destrezas técnicas de baja complejidad que se intentaron cubrir con programas de enseñanza por correspondencia.

El mundo se ha transformado y con él se han transformado los hombres y mujeres que lo habitan, sus productos materiales y simbólicos, sus formas de comunicación y entendimiento. Es imposible negar hoy las tendencias irreversibles de la globalización en cuya amplia trama habrá de incluirse la globalización del conocimiento. De igual manera es imposible desatender las demandas de transformación de las instituciones educativas, en particular de las universidades, debido a una sociedad cada vez más dependiente del conocimiento.

Se ha podido analizar, contrastar y comparar las ofertas educativas de algunas universidades de América Latina como el Instituto Tecnológico de Monterrey (TEC) de México, Universidad de Costa Rica, Universidad del Mar del Plata de Argentina, Universidad Javeriana de Colombia, entre otras, las que haciendo la salvedad del contexto socioeconómico en el que se encuentran, tienen como denominador común el brindar la mejor atención a su cliente, el alumno.

En muchos de los casos el campo de acción de estas instituciones es limitado, sobre todo para acceder a las áreas rurales donde existen desigualdades y carencias como desempleo, pobreza, exclusión social, migraciones, analfabetismo, entre otros. Sin embargo, las muchas modalidades de educación superior ofrecidas por estas universidades constituyen alternativas para acceder a la información, procesarla, enriquecerla y convertirla en conocimiento, que se constituye en el capital intelectual de las organizaciones de hoy.

\section{VISIÓN CRÍTICA}

Las diferencias económicas existentes actualmente en la mayoría de los países del mundo limitan las posibilidades de desarrollo científico, técnico y cultural de los distintos grupos humanos de cada sociedad. Se requiere, por lo tanto, de un esfuerzo especial para lograr la realización plena y multifacética del ser humano.

La educación a distancia es una posibilidad real y eficaz para mejorar la transferencia de infor- mación y lograr el aprendizaje de los individuos a bajo costo. Gracias a ella se reducen los obstáculos que representan el tiempo y el espacio; además de utilizarse métodos, técnicas y recursos que elevan la productividad y la flexibilidad del proceso de enseñanza-aprendizaje. La utilización de tecnologías como la radio, televisión, el video, la cinta de audio, los sistemas informáticos de complejidad variable y los software interactivos, constituyen nuevas muestras de la vigencia y procedencia de los principios que sustentan la educación para todos, el aprender a aprender, la enseñanza-aprendizaje personalizada, la imprescindible educación para toda la vida. El empleo de métodos innovadores favorece un mayor protagonismo de los alumnos en su propio aprendizaje a través de la educación a distancia.

Sin embargo, al revisar las bondades de los cursos a distancia que ofrecen las distintas instituciones educativas, encontramos notorias diferencias: importantes facilidades para el estudio que contribuyen a la construcción de las capacidades del individuo pero, al mismo tiempo, se aprecian muchas limitaciones que procederemos a enumerar y explicar.

- Limitaciones tecnológicas. El desarrollo de redes telemáticas es el resultado de los logros alcanzados en materia de telecomunicaciones. Gracias a que hoy en día contamos con un mayor ancho de banda, los datos pueden transmitirse con una alta calidad y rapidez. El uso de tecnologías de cable coaxial, del par trenzado y de la fibra óptica y los satélites, ha permitido cubrir adecuadamente enormes áreas geográficas.

No obstante los costos siguen siendo altos para muchas familias. Adicionalmente existen zonas más alejadas de las ciudades que no cuentan con fluido eléctrico (elemento clave para acceder a las nuevas tecnologías), mientras que los que lo tienen necesitan una gran capacitación en el manejo de computadoras y sus programas básicos a fin de poder realizar trabajos en red.

- Limitaciones de los educandos. La educación a distancia se va imponiendo día a día en nuestro continente y específicamente en nuestro país; sin embargo esta modalidad educativa tiene que vencer muchas trabas, además de cambiar el modo de vida de las personas. Estudiar a distancia significa lograr autonomía y una férrea disciplina personal 
de estudiar a solas, estableciendo horarios y espacios según los estilos de aprendizaje de cada uno, logrando la automotivación continua y perseverancia para alcanzar los objetivos trazados.

La educación en línea requiere tomar decisiones personales para gestionar el tiempo y espacio y asumir responsablemente el autoaprendizaje, haciendo el compromiso de ser, estar y participar en los actos educativos que se dan en la formación on line asumiendo un rol activo, participativo

- Limitaciones metodológicas. El contacto individualizado e interactivo entre los miembros de la comunidad de aprendizaje (estudiantes, docentes y tutores), no se concretiza en su totalidad por el hecho de que el alumno no está familiarizado con el uso de la tecnología, no se siente en confianza en interactuar, pudiéndose apreciar bloqueos al momento de manejar los medios tecnológicos en sesiones sincrónicas y asincrónicas que se dan en línea, tales como el chat, forum, conferencias virtuales, e-mail.

Los contactos a través del chat son enriquecedores y mucho más la participación en los forum donde se plasman las ideas y se intercambian puntos de vista.

\section{PROPUESTAS Y CONSIDERACIONES}

- De las instituciones. Las universidades han de realizar alianzas o convenios con otras instituciones haciendo más ricos y variados sus ofrecimientos educativos, mejorando sus tarifas, requerimientos de admisión, proyectos educativos, innovaciones educativas e investigación educacional.

- De la enseñanza. La educación que promueve el campus virtual ha de tener como valor agregado el de una formación de calidad, que sea homologable con la enseñanza presencial. La educación a distancia permite conectar a estudiantes, profesores y tutores de distintas nacionalidades y regiones, lográndose un enriquecedor intercambio multicultural que influirá positivamente en el crecimiento personal y profesional de lo sujetos, así como en el desarrollo institucio-nal, revalorando la diversidad e interculturalidad de los pueblos.

- De los tutores. La educación a distancia ha de revalorar el papel del docente cuyo rol es clave en la educación on line, además de hacer patente la innegable necesidad de su constante capacitación y actualización.

- De los educandos. La educación a distancia ha de hacer de los educandos los gestores de su cambio personal: la toma de decisiones en el autoaprendizaje, automotivación, disciplina en el manejo del espacio, tiempo y técnicas de estudio; que permitirán analizar, ordenar, clasificar, representar, mejorar, interpretar y evaluar la información on line, para llegar a la mejora continua y lograr la eficiencia, eficacia y productividad de la institución.

\section{CONCLUSIONES}

1. La formación que promueven las instituciones de educación a distancia, en este caso las universidades, debe proporcionar un valor agregado a su oferta educativa, siendo fundamental una formación de calidad homologable con la enseñanza presencial. Debe permitirle al alumno una adquisición de conocimientos generales y específicos sobre diversos enfoques de desarrollo humano, gestión de la información, uso de herramientas tecnológicas, participación en redes de trabajo e intercambio de ideas en un entorno virtual e interacción multicultural, concepto que se entiende como el de pertenencia a un grupo de personas con distinto origen geográfico y cultural enriquecido considerablemente por los proceso de comunicación y aprendizaje de estudiantes, docentes y tutores.

2. Las regiones deben tener una política conjunta de mitigar gastos de deuda externa para lograr la conectividad, mejorando la infraestructura y accesibilidad para que se beneficie toda la población a través de la educación a distancia, abaratándose los costos de equipos de computación y las tarifas de conexión.

3. Realizar campañas, convenios y conformar redes institucionales de cooperación real entre universidades experimentadas y no experimentadas para ofrecer educación virtual. Estas redes estarán constituidas por equipos multidisciplinarios de especialistas para evaluar los estándares de diseño tecnológicos y pedagógico ofrecidos por la plataforma virtual.

4. Conformar grupos regionales y multidisciplinarios de investigación en aspectos tecnológicos y de infraestructura, que realicen 
diagnósticos sobre necesidades virtuales y áreas de especialización; estudios para el diseño de políticas educativas, nacionales y regionales en materia de educación virtual; estudios de legislación y normatividad; fundamentos conceptuales; modelos educativos; diseño instruccional; desarrollo de contenidos de e-learning; evaluación y programación de plataformas en base a software libre y aplicativos, así como la elaboración de base de datos para bibliotecas virtuales.

5. Buscar el apoyo de la cooperación internacional para financiar proyectos.

6. Propulsar foros y debates regionales que proporcionen nuevos conocimientos y enriquezcan la interacción.

7. Finalmente, se ha de consolidar el cambio de nuestras comunidades y pueblos incorporando las tecnologías de información y la comunicación en la educación a distancia, supri- miendo las barreras espacio-temporales, consiguiendo la mejora cuantitativa y cualitativa de los procesos de enseñanza-aprendizaje en línea, que el hombre del presente siglo demanda.

\section{BIBLIOGRAFÍA}

The Institute for Highter Education Policy. Quality on the Line. April, 2000.

Barker, K. Quality guidelines for technology-assisted distance education.

Lima De Moura, Sheila y Leite, Ligia Silvia. Indicadores de calidad de los cursos virtuales.

Facundo D., A. H. Educación virtual en América Latina y el Caribe: características y tendencias (http:/ /www. educorp. futuro.usp.br/publicacoes/ eduvirtualunesco.pdf), 2002. 